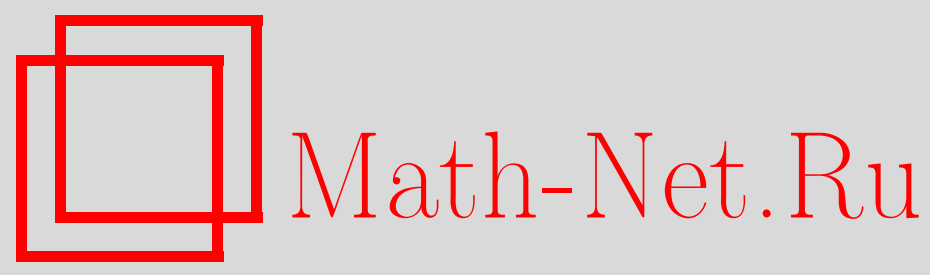

Б. А. Кац, Интегрирование по фрактальной кривой и задача о скачке, Матем. заметки, 1998, том 64, выпуск 4, 549557

DOI: https://doi.org/10.4213/mzm1429

Использование Общероссийского математического портала Math-Net.Ru подразумевает, что вы прочитали и согласны с пользовательским соглашением http://www . mathnet.ru/rus/agreement

Параметры загрузки:

IP : 54.237 .59 .107

26 апреля 2023 г., 15:15:08

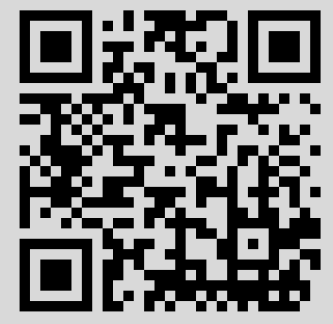




\section{ИНТЕГРИРОВАНИЕ ПО ФРАКТАЛЬНОЙ КРИВОЙ \\ И ЗАДАЧА О СКАЧКЕ}

\section{Б. А. Кац}

В статье дается определение интегрирования, т.е. обобщения функционала вида

$$
u(z) \mapsto \int_{\Gamma} f(z) u(z) d z
$$

на случай, когда Г есть фрактальная кривая на комплексной плоскости, а $f(z)$ - заданная на ней функция (плотность интегрирования). Исследуются вопросы существования и единственности интегрирования с заданной плотностью.

Библиографоия: 20 названий.

1. Введение. $\mathrm{K}$ настоящему времени опубликован ряд работ, посвященных определению и исследованию интеграла $\int_{\Gamma} u(z) d z$ по фрактальному контуру $\Gamma$ (см. [1]-[5]). Почти во всех этих работах используются идеи геометрической теории интеграла. В данной заметке мы определяем интеграл иначе.

Пусть Г есть заданная на комплексной плоскости $\mathbb{C}$ спрямляемая кривая. Если определенная на ней плотность $f(z)$ интегрируема по мере $d s$, то функционал

$$
u(z) \mapsto \int_{\Gamma} f(z) u(z) d z
$$

корректно определен на $C(\Gamma)$ и, тем более, на $C^{\infty}(\mathbb{C})$, т.е. его можно рассматривать как обобщенную функцию на комплексной плоскости с компактным носителем на кривой $Г$.

Определим интегрирование по фрактальной кривой Г как обобщенную функцию с носителем на этой кривой, удовлетворяющую ряду аксиом, позволяющих трактовать плотность интегрирования как обычную функцию на Г. Мы покажем, что на спрямляемой кривой такому определению удовлетворяют лиш обобщенные функции вида (1). Для фрактальных кривых выясним условия существования интегрирования с заданной плотностью и обсудим вопросы единственности.

Работа выполнена при поддержке Российского фонда фундаментальных исследований, грант № 95-01-00674. 
2. Определения интегрирований. Если $\varphi$ есть обобщенная функция на комплексной плоскости с компактным носителем, то определим голоморфную вне ее носителя функцию $k_{\varphi}(z)=(2 \pi i)^{-1} \varphi\left((\cdot-z)^{-1}\right)$, которую будем называть ее преобразованием Коши. Если носитель $\varphi$ имеет плоскую меру 0 и функция $k_{\varphi}(z)$ локально интегрируема на комплексной плоскости, то отождествим ее с распределением

$$
u \mapsto \iint_{\mathbb{C}} k_{\varphi}(z) u(z) d x d y
$$

где $z=x+i y$ и $u \in C_{0}^{\infty}(\mathbb{C})$.

Далее, пусть для обобщенной функции $\varphi$ с компактным носителем определена свертка $\varphi * E$, где $E=(\pi z)^{-1}$ (см., например, [6]). Нетрудно показать, что вне носителя $\varphi$ эта свертка совпадает с распределением $-2 i k_{\varphi}$. Поэтому носитель суммы

$$
\varphi * E+2 i k_{\varphi}
$$

лежит в носителе $\varphi$. Вообще говоря, сумма (2) может быть отличной от 0 (например, если $\varphi$ есть производная по $\bar{z}$ от $\delta_{0}$, то эта сумма равна $\left.\delta_{0}\right)$. Однако, она равна 0 для распределения (1) с носителем на спрямляемой кривой Г. Кроме того, преобразование Коши такого распределения есть интеграл типа Коши

$$
k(z)=\frac{1}{2 \pi i} \int_{\Gamma} \frac{f(t) d t}{t-z} .
$$

Если Г есть замкнутая кусочно-гладкая кривая, а функция $f(t)$ удовлетворяет условию Гёльдера

$$
\sup \left\{\frac{\left|f\left(t^{\prime}\right)-f\left(t^{\prime \prime}\right)\right|}{\left|t^{\prime}-t^{\prime \prime}\right|^{\nu}}: t^{\prime}, t^{\prime \prime} \in \Gamma, t^{\prime} \neq t^{\prime \prime}\right\}=h_{\nu}(f, \Gamma)<\infty
$$

(всюду далее множество всех таких функций обозначаем через $H_{\nu}(\Gamma), 0<\nu \leqslant 1$ ), то в каждой точке $t \in \Gamma$ функция $k(z)$ имеет предельные значения с обеих сторон, причем разность этих предельных значений

$$
k^{+}(t)-k^{-}(t)=f(t)
$$

Здесь $k^{+}(t)$ есть предельное значение $k(z)$ при приближении $z$ к $t$ из ограниченной кривой $\Gamma$ конечной области $D^{+}$, a $k^{-}(t)$ - при приближении из дополняющей $D^{+}$до плоскости $\mathbb{C}$ бесконечной области $D^{-}$. На негладкой спрямляемой кривой соотношение $(3)$ справедливо при дополнительном условии

$$
\nu>\frac{1}{2}
$$

(см. [7]). На этом основании мы можем определить интегрирование по произвольной (вообще говоря, неспрямляемой) кривой следующим образом. 
ОПРЕДЕЛЕниЕ 1. Пусть Г есть простая замкнутая кривая. Распределение $\varphi$ с носителем на этой кривой называется непрерывным интегрированием, если для него сумма (2) равна 0 тождественно, причем преобразование Коши $k_{\varphi}$ представляет собой функцию непрерьвную в замыканиях областей $D^{+}$и $D^{-}$. Разность (3) граничных значений этой функщии есть плотность интегрирования $\varphi$.

При нарушении условия (4) интеграл типа Коши по негладкой спрямляемой кривой уже не обязан иметь предельные значения во всех точках кривой, однако почти всюду на ней (относительно меры $d s$ ) он имеет некасательные предельные значения с обеих сторон, и они по-прежнему связаны соотношением (3). То же относится к интегралам типа Коши по кусочно-гладким кривьп с непрерывной (но негёльдеровой) и разрьвной (интегрируемой по $d s$ ) плотностью. Для неспрямляемой кривой теряет смысл понятие некасательных пределов и отсутствует мера $d s$. Однако, здесь мы можем воспользоваться результатами А. Йонсона и Х. Валлина. Они рассмотрели [8] так называемые $d$-множества. Кривая Г является $d$-множсеством, если существуют постоянные $c>1, r_{0}>0$ такие, что для любого $z \in \Gamma$ и любого положительного $r \leqslant r_{0}$ справедлива оценка

$$
c^{-1} r^{d} \leqslant m_{d}(\Gamma \cap B(z, r)) \leqslant c r^{d}
$$

где $B(z, r)$ - круг радиуса $r$ с центром в точке $z, 1<d<2$, и $m_{d}$ - мера Хаусдорфа размерности $d$. Очевидно, всякое $d$-множество имеет размерность Хаусдорфа $d$ (определение меры и размерности Хаусдорфа см., например, в [9]).

С точки зрения прикладной математики наиболее интересны неспрямляемые кривые, обладающие свойством самоподобия (см., например, [10], [11]). Кривая Г назьвается самоподобной, если она содержит дугу $\Gamma_{0}$ такую, что любая другая дуга $\Gamma^{\prime} \subset \Gamma$ имеет часть $\Gamma^{\prime \prime} \subset \Gamma^{\prime}$ подобную $\Gamma_{0}$. Наиболее известным примером неспрямляемой замкнутой самоподобной кривой является так назьваемая снежинка фон Коха. Она является гранищей области $D=\bigcup_{n=0}^{\infty} D_{n}$, где $D_{0}$ - правильньй треугольник со стороной длины 1 , а $D_{n}$ - многоугольник со сторонами длины $3^{-n}$, полученный из многоугольника $D_{n-1}$ присоединением к средней трети каждой его стороны правильного треугольника со стороной $3^{-n}$, лежащего вне $D_{n-1}$. Снежинка Коха имеет клеточную размерность $d=\log _{3} 4$ (определение клеточной размерности см., например, в [10], [11]). Клеточная размерность и размерность Хаусдорфа самоподобного множества совпадают; кроме того, любой самоподобньй фрактал клеточной размерности $d$ является $d$-множеством (см., например, [12]).

П. У. Джонс [13] ввел понятие $(\varepsilon, \delta)$-множества. Область $D$ является $(\varepsilon, \delta)$-множеством, если любые две точки $x, y$ этой области такие, что $|x-y|<\delta$, можно соединить в ней спрямляемой дугой $\gamma$ длины не более $|x-y| / \varepsilon$ так, что

$$
\operatorname{dist}(z, \Gamma) \geqslant \frac{\varepsilon|x-z| \cdot|z-y|}{|x-y|}
$$

для любой точки $z \in \gamma$. Если Г есть замкнутая самоподобная фрактальная кривая, то обе области $D^{+}, D^{-}$являются $(\varepsilon, \delta)$-множествами для некоторых $\varepsilon$ и $\delta$. В работе [14] $\mathrm{X}$. Валлин получил следующий результат.

ТеоремА. Если кривая Г является $d$-множсеством, $1<d<2$, область $D^{+}$ (или $\left.D^{-}\right)$есть $(\varepsilon, \delta)$-множество для некоторых $\varepsilon, \delta$, а заданная в области $D^{+}$ 
(соответственно в $D^{-}$) функиия $k(z)$ принадлежит пространству Соболева $W_{1}^{p}$, $p>1$, то для $m_{d}$-почти всех $t \in \Gamma$ существуют предель

$$
k^{+}(t)=\lim _{r \rightarrow 0} \frac{1}{m_{2}\left(D^{+}(t, r)\right)} \iint_{D^{+}(t, r)} k(x+i y) d x d y
$$

соответственно

$$
k^{-}(t)=\lim _{r \rightarrow 0} \frac{1}{m_{2}\left(D^{-}(t, r)\right)} \iint_{D^{-}(t, r)} k(x+i y) d x d y
$$

где $D^{ \pm}(t, r)=D^{ \pm} \cap B(t, r)$, а $m_{2}$ - обычная плоская мера Лебега.

Как обычно, под $W_{1}^{p}$ мы понимаем пространство всех тех $L^{p}\left(m_{2}\right)$-функций, чьи первые обобщенные производные также являются $L^{p}\left(m_{2}\right)$ - функциями. В случае бесконечной области $D^{-}$здесь подразумевается интегрируемость по любой ее конечной части.

Для самоподобного фрактала размерности $d, 1<d<2$, существуют оба предела $(5),(6)$. Их можно рассматривать как граничные значения соответствующих функций. Это приводит к следующему определению.

ОПРЕДЕЛЕниЕ 2. Пусть Г есть простая замкнутая самоподобная фрактальная кривая размерности $d, 1<d<2$. Распределение $\varphi$ с носителем на этой кривой называется разрывным интегрированием, если для него сумма (2) равна 0 тождественно, причем преобразование Коши $k_{\varphi}$ представляет собой функцию, сужения которой на области $D^{+}$и $D^{-}$принадлежат пространствам $W_{1}^{p}, p>1$. Определенная $m_{d}$-почти всюду на $Г$ разность (3) граничных значений $(5),(6)$ этой функции есть плотность интегрирования $\varphi$.

Теперь мы рассмотрим вопрос о существовании и единственности непрерьвных и разрьвных интегрирований с заданной плотностью.

3. Существование интегрирований. Поскольку $\varphi * E+2 i k_{\varphi}=0$, то

$$
\varphi=-2 i \frac{\partial k_{\varphi}}{\partial \bar{z}}
$$

т.e.

$$
\varphi(u)=-\iint \frac{\partial u}{\partial \bar{z}} k_{\varphi}(z) d z d \bar{z}, \quad u \in C_{0}^{\infty}
$$

Таким образом, интегрирование однозначно восстанавливается по своему преобразованию Коши, и вопрос о существовании интегрирований с заданной плотностью есть вопрос о разрешимости задачи о скачке (3) в нужном классе функций.

Пусть $f \in H_{\nu}(\Gamma)$. Обозначим через $f^{w}(z)$ ее продолжение Уитни (см. [6]). Напомним, что функция $f^{w}$ удовлетворяет условию Гёльдера с показателем $\nu$ во всей комплексной плоскости, совпадает с $f$ на $\Gamma$, а вне $Г$ имеет частные производные всех порядков. В [15] показано, что ее первые производные интегрируемы в $D^{+}$в любой степени меньшей $(2-d) /(1-\nu)$, где $d-$ клеточная размерность $Г$. При условии

$$
\nu>d-1
$$


эта величина превосходит 1, и мы можем рассмотреть функцию

$$
k(z)=f^{w}(z) \chi(z)-\frac{1}{2 \pi i} \iint_{D^{+}} \frac{\partial f^{w}}{\partial \bar{t}} \frac{d t d \bar{t}}{t-z}
$$

где $\chi$ - характеристическая функция области $D^{+}$. При условии

$$
\nu>\frac{d}{2}
$$

производная $\partial f^{w} / \partial \bar{t}$ интегрируема в степени больше 2 , и интегральньй член (9) представляет собой непрерывную функцию (см., например, [16]). Таким образом, при условии (10) функция (9) голоморфна в $D^{+}$и в $D^{-}$, непрерьвна в замьканиях этих областей и удовлетворяет краевому условию (3) всюду на Г. Подставив ее в правую часть (7) в качестве $k_{\varphi}$, мы получим непрерывное интегрирование с плотностью $f$. Итак, справедлива

ТЕОРема 1. Пусть на простой замкнутой кривой Г клеточной размерности $d$ задана функиия $f \in H_{\nu}(\Gamma)$. При условии (10) существует непрерывное интегрирование по Г с плотностью $f$.

Из построений [15] следует, что условие (10) неулучшаемо в классе всех кривых размерности $d$. Однако, для самоподобных фрактальных кривых оно может быть ослаблено. Недавно Т. Е. Объедков [17] показал, что на таких кривых задача о скачке (3) имеет непрерьвные решения при более слабом условии (8). Из этого результата следует

ТЕОРема 2. Пусть на простой замкнутой самоподобной фрактальной кривой Г размерности d задана функиия $f \in H_{\nu}(\Gamma)$. При условии (8) существует непрерывное интегрирование по Г с плотностью $f$.

Теперь рассмотрим разрьвные интегрирования. Непосредственное дифференцирование показывает, что производная $k^{\prime}$ функции (9) интегрируема в любой конечной части плоскости в той же степени, что и производные продолжения Уитни $\partial f^{w} / \partial x, \partial f^{w} / \partial y$. Таким образом, при условии (8) сужения функции (9) на области $D^{+}$и $D^{-}$принадлежат пространствам Соболева $W_{1}^{p}$ с некоторым $p>1$, и порождаемое этой функцией интегрирование подпадает под определение 2. Иначе говоря, при условиях теоремы 2 существует разрывное интегрирование по кривой $Г$ с плотностью $f$, которое одновременно является и непрерьвным интегрированием. Разрьвные интегрирования (не являющиеся, вообще говоря, непрерьвными) существуют и при более слабых ограничениях на плотность. Согласно [8] пространства Бесова $B_{\beta}^{p, p}$ можно определить на $d$-множестве $\Gamma$ следуюшим образом. Пусть $0<\beta<1, p>1$ и $\mu$ есть сужение меры Хаусдорфа $m_{d}$ на замкнутое $d$-множество $\Gamma$. Для каждого натурального $n$ построим разбиение $M_{n}$ комплексной плоскости на квадраты со стороной $2^{-n}$. Функция $f \in L^{p}(\mu)$ принадлежит пространству Бесова $B_{\beta}^{p, p}(\Gamma)$, если для каждого натурального $n$ можно указать постоянную на каждом из квадратов разбиения $M_{n}$ функцию $f_{n}$ такую, что ряд

$$
\sum_{n=1}^{\infty}\left(2^{n \beta}\left\|f-f_{n}\right\|_{L^{p}(\mu)}\right)^{p}
$$


сходится. Согласно [14] при

$$
\beta=1-\frac{2-d}{p}
$$

всякая функция $f \in B_{\beta}^{p, p}(\Gamma)$ может быть продолжена до функции $F \in W_{1}^{p}(\mathbb{C})$ так, что предел

$$
F^{*}(t)=\lim _{r \rightarrow 0} \frac{1}{\pi r^{2}} \iint_{B(t, r)} F(x+i y) d x d y
$$

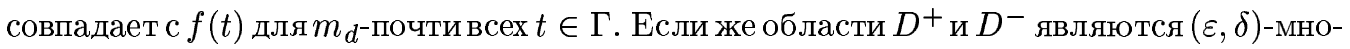
жествами, то (см. [14])

$$
F^{*}(t)=F^{+}(t)=F^{-}(t)
$$

$m_{d}$-почти всюду на $\Gamma$. Здесь $F^{ \pm}(t)$ - граничные значения сужений $F$ на $D^{ \pm}$, вычисляемые по формулам (5), (6). Подставив продолжение $F$ вместо продолжения Уитни $f^{w}$ в формулу (9), мы приходим к следующему результату.

ТЕОРемА 3. Пусть на простой замкнутой самоподобной фрактальной кривой Г размерности $d$ задана функиия $f \in B_{\beta}^{p, p}(\Gamma)$, где $p>1,1<d<2$, а $\beta$ определяется равенством (11). Тогда существует разрывное интегрирование по Г с плотносmвю $f$.

4. Единственность интегрирований. Разность преобразований Коши двух непрерьвных интегрирований с одинаковыми плотностями есть непрерьвная в $\mathbb{C}$ и голоморфная в $\mathbb{C} \backslash \Gamma$ функция. Согласно теореме Пенлеве (см., например, [18]) такая функция голоморфна на $\Gamma$, если эта кривая спрямляема. Тогда соответствующие непрерьвные интегрирования совпадают. Таким образом, справедлива

ТЕОРемА 4. На простой замкнутой спрямляемой кривой существует не более одного непрерывного интегрирования с заданной плотностью. В частности, единственное непрерывное интегрирование с плотностью $f \in H_{\nu}(\Gamma), \nu>1 / 2$, имеет вид (1).

Если кривая Г неспрямляема и имеет размерность Хаусдорфа $d_{H}>1$, то существуют непрерывные в $\mathbb{C}$ и голоморфные в $\mathbb{C} \backslash \Gamma$ функции, не являюшиеся голоморфными в точках кривой $Г$. В частности, это относится к любому самоподобному фракталу размерности $d>1$. Можно сказать, что на таком фрактале существуют нетривиальные интегрирования с нулевой плотностью, и непрерывное интегрирование с заданной плотностью по такому фракталу заведомо неединственно. Преобразования Коши интегрирований с нулевой плотностью (т.е. непрерьвные в $\mathbb{C}$ и голоморфные в $\mathbb{C} \backslash \Gamma$ функции, исчезающие в точке $\infty$ ) образуют алгебру. Как показанов [19], эта алгебра равномерна (речь идет об алгебре $A(\overline{\mathbb{C}}, \overline{\mathbb{C}} \backslash \Gamma$ ) в обозначениях [19]). Все известные автору примеры нетривиальных элементов этой алгебры (см. [18], [20]) имеют вид

$$
\int_{\Gamma} \frac{d \mu(t)}{t-z}
$$

где $\mu$ есть сужение меры Хаусдорфа $m_{d}$ на $Г$ или на некоторое подмножество $\Gamma$, так что соответствующие интегрирования суть конечные меры на Г. Было бы интересно выяснить, существуют ли интегрирования с нулевыми плотностями, не являющиеся мерами. Автор полагает, что на самоподобных фракталах таких интегрирований нет. 
Теперь мы перейдем к разрьвным интегрированиям. На первьй взгляд класс таких интегрирований может показаться более обширным, чем множество непрерывных интегрирований с той же плотностью. Однако, нетривиальных интегрирований с нулевой плотностью в этом классе нет.

ТЕОРема 5. На простой замкнутой самоподобной фрактальной кривой размерности $d, 1<d<2$, существует не более одного разрывного интегрирования $c$ заданной плотностью.

ДокАЗАтЕльство. Разность преобразований Коши двух разрывных интегрирований с одинаковой плотностью есть голоморфная в $\overline{\mathbb{C}} \backslash \Gamma$ функция $g(z)$, сужения которой на области $D^{ \pm}$принадлежат пространствам Соболева $W_{1}^{p}$, а граничные значения $(5),(6) m_{d}$-почти всюду на $\Gamma$ связаны соотношением $g^{+}(t)=g^{-}(t)$. Поскольку $D^{-}$есть $(\varepsilon, \delta)$-множество, то [13] существует функция $h \in W_{1}^{p}(\mathbb{C})$, совпадающая с $g(z)$ в $D^{-}$, причем $m_{d}$-почти всюду на $\Gamma$ ее лебеговы значения $h^{*}(t)$ (см. $\left.(12)\right)$ совпадают с $g^{ \pm}(t)$. Разность $g(z)-h(z)=f(z)$ равна 0 в $D^{-}$, а ее сужение на $D^{+}$принадлежит пространству $W_{1}^{p}\left(D^{+}\right)$и имеет нулевые граничные значения (5). Тогда [14] $\left.f\right|_{D^{+}}$есть предел в $W_{1}^{p}\left(D^{+}\right)$некоторой последовательности функций $f_{n} \in C_{0}^{\infty}\left(D^{+}\right), n=1,2, \ldots$ Продолжим каждую из функций $f_{n}$ на всю плоскость нулем и вычислим предел полученной последовательности в $W_{1}^{p}(\mathbb{C})$. Очевидно, этот предел совпадает с $f$. Таким образом, $f \in W_{1}^{p}(\mathbb{C}), g=h+f \in W_{1}^{p}(\mathbb{C})$ и обобщенная производная $g$ по $\bar{z}$ есть обычная $L^{p}$-функция. Поскольку вне $Г$ она равна 0 и плоская мера $Г$ есть 0 (это следует из условия $d<2$ ), то $\partial g / \partial \bar{z}=0$ в смысле теории обобщенных функций. Теорема доказана.

В частности, при $1>\nu>d-1>0$ на самоподобной фрактальной кривой $Г$ существует бесконечное множество непрерьвных интегрирований с заданной плотностью $f \in H_{\nu}(\Gamma)$, но лиш одно из них является разрывным. Преобразование Коши этого интегрирования определяется формулой (9), а преобразования Коши остальных непрерьвных интегрирований с этой плотностью не попадают в пространства Соболева.

5. Интегрирования по разомкнутой дуге. Пусть теперь Г есть ориентированная простая дуга с началом в точке $a$ и концом в точке $b \neq a$. Дуга $\Gamma$ не разбивает комплексную плоскость на области $D^{+}, D^{-}$, и под граничными значениями $k^{+}(t), k^{-}(t)$ мы должны понимать предельные значения $k(z)$ при приближении $z$ к $t$ слева и справа соответственно. В точках $a, b$ такое определение теряет смысл. Кроме того, интеграл типа Коши по разомкнутой гладкой дуге имеет логарифмические особенности на ее концах. В случае негладкой спрямляемой дуги эти особенности могут быть сколь угодно сильными. Поэтому определение непрерывного интегрирования в случае разомкнутой дуги должно быть изменено следующим образом.

ОПРЕДЕЛЕНИЕ 3. Пусть Г есть простая ориентированная дуга с началом $a$ и концом $b$. Распределение $\varphi$ с носителем на этой дуге назьвается непрерывным интегрированием, если его преобразование Коши $k_{\varphi}$ представляет собой интегрируемую в любой конечной части плоскости функцию, имеющую в каждой точке $t \in \Gamma \backslash\{a, b\}$ непрерывные предельные значения с обеих сторон $k_{\varphi}^{+}(t), k_{\varphi}^{-}(t)$, а сумма (2) равна 0 тождественно. Разность граничных значений функции $k_{\varphi}$ есть плотность интегрирования $\varphi$. Она определена при $t \in \Gamma \backslash\{a, b\}$.

Формула (9), использовавшаяся при доказательстве существования непрерывных ин- 
тегрирований по замкнутой кривой, приобретает здесь иной вид:

$$
k(z)=f^{w}(z) \chi_{\Gamma}(z)-\frac{1}{2 \pi i} \iint_{\mathbb{C}} \frac{\partial f^{w}}{\partial \bar{t}} \chi_{\Gamma}(z) \frac{d t d \bar{t}}{t-z} .
$$

Здесь продолжение $f^{w}$ должно иметь компактньй носитель, а место характеристической функции $\chi$ занимает однозначная ветвь логарифма

$$
\chi_{\Gamma}(z)=\frac{1}{2 \pi i} \ln \frac{z-b}{z-a} \text {, }
$$

выделенная посредством разреза по Г и условия $\chi_{\Gamma}(\infty)=0$. Логарифм $(14)$ имеет единичньй скачок на дуге Г. Если он имеет на концах $Г$ логарифмические особенности (или интегрируем в любой степени по любой конечной части плоскости), то все оценки интеграла из (13) сохраняют силу. Поэтому результаты теорем 1, 2, 4 справедливы для разомкнутой дуги в виде следующего утверждения.

ТЕОрема 6. Пусть на простой разомкнутой дуге Г клеточной размерности $d$ задана функиия $f \in H_{\nu}(\Gamma)$, а логарифм (14) интегрируем в любой конечной положительной степени по любой конечной части плоскости. Тогда справедливь следующие утверэсдени:

а) при условии (10) существует непрерывное интегрирование по Г с плотностью $f$

б) если дуга Г является самоподобным фракталом размерности $d<2$, то это интегрирование существует при более слабом условии (8);

в) если любая собственная (т.е. не содержащая кониов $a, b)$ часть Г спрямляема, то на ней существует не более одного непрерывного интегрирования с заданной плотностью.

Теперь перейдем к разрьвным интегрированиям. Точки $a$ и $b$ можно соединить дугой $\gamma$ так, что $\Gamma \cap \gamma=\{a, b\}$ и каждая собственная часть $\gamma$ является гладкой. Объединение $\Gamma \cup \gamma$ есть замкнутая кривая, разбивающая комплексную плоскость на конечную область $D^{+}$и бесконечную $D^{-}$. Использование этих областей позволяет перенести определение разрьвных интегрирований на разомкнутые дуги почти без изменений.

ОПРЕДЕЛЕниЕ 4. Пусть Г есть простая разомкнутая самоподобная фрактальная дуга размерности $d, 1<d<2$. Распределение $\varphi$ с носителем на этой дуге назьвается $р а$ аз- $^{-}$ рывным интегрированием, если для него сумма (2) равна 0 тождественно, причем преобразование Коши $k_{\varphi}$ представляет собой функцию, сужения которой на области $D^{+}$

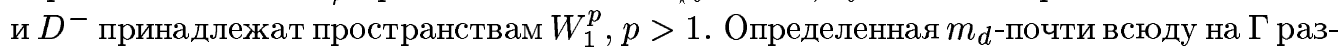
ность (3) граничных значений $(5),(6)$ этой функции есть плотность интегрирования $\varphi$.

Нетрудно показать, что определяемьй таким образом класс распределений не зависит от произвола в выборе замыкающей дуги $\gamma$ (аналогичным образом мы могли определить и непрерывные интегрирования на разомкнутой дуге). Разность логарифмов $\chi_{\Gamma}(z)-\chi_{\gamma}(z)$ представляет собой характеристическую функцию области $D^{+}$(возможно, со зна́ком “-”), так что эти логарифмы имеют одинаковые особенности в точках $a, b$. Это позволяет перенести результаты теорем 3 и 5 на разомкнутые дуги в виде следующего утверждения. 
Tеорема 7. Пусть Г есть простая самоподобная фрактальная дуга размерности $d, 1<d<2$, для которой логарифм (14) интегрируем в любой положительной степени по любой конечной части комплексной плоскости. Если на Г задана функиия $f \in B_{\beta}^{p, p}(\Gamma)$, где $p>1$, а $\beta$ определяется равенством (11), то существует единственное разрывное интегрирование по Г с плотностью $f$.

\section{СПИСОК ЦИТИРОВАННОЙ ЛИТЕРАТУРЫ}

[1] Кац Б. А. Задача о скачке и интеграл по неспрямляемой кривой // Изв. вузов. Матем. 1987. № 5. C. $49-57$.

[2] Harrison J., Norton A. Geometric integration on fractal curves in the plane // Indiana Univ. Math. J. 1991. V. 40. № 2. P. 567-594.

[3] Harrison J., Norton A. The Gauss-Green theorem for fractal boundaries // Duke Math. J. 1992. V. 67. № 3. P. 575-586.

[4] Кац Б. А. Об интегрировании по неспрямляемой кривой // Вопросы матем., механики сплошных сред и применения матем. методов в строительстве. М.: МИСИ, 1992. С. 63-69.

[5] Harrison J. Stokes' theorem for nonsmooth chains // Bull. Amer. Math. Soc. 1993. V. 29. № 2. P. 235-242.

[6] Хёрмандер Л. Анализ линейных дифференциальных операторов с частными производными. Т. 1. М.: Мир, 1986.

[7] Дынькин Е. М. Гладкость интеграла типа Коши // Записки научн. семин. ЛОМИ. 1979. T. 92 . C. $115-133$.

[8] Jonsson A., Wallin H. Function spaces on subsets of $\mathbb{R}^{n} / /$ Math. Rep. V. 2. Part 1. London: Harwood Acad. Publ., 1984.

[9] Карлесон Л. Избранные проблемы теории исключительных множеств. М.: Мир, 1971.

[10] Mandelbrot B. B. The Fractal Geometry of Nature. San Francisco: Freeman, 1982.

[11] Федер Е. Фракталы. М.: Мир, 1991.

[12] Väsäla J., Vuorinen M., Wallin H. Thick Sets and Quasi-symmetric Maps. Reports of Dept. of Math. Preprint № 2 (February, 1992). Espoo: Univ. of Helsinki, 1992.

[13] Jones P.W. Quasiconformal mappings and extendability of functions in Sobolev spaces // Acta Math. 1981. V. 147. №1-2. P. 71-88.

[14] Wallin H. The trace to the boundary of Sobolev space on a snowflake // Manuscripta Math. 1991. V. 73. P. 117-125.

[15] Кац Б. А. Задача Римана на замкнутой жордановой кривой // Изв. вузов. Матем. 1983. № 4. C. $68-80$.

[16] Векуа И.Н. Обобщенные аналитические функции. М.: Наука, 1988.

[17] Объедков Т. Е. Применение нестандартного анализа к теории интегрирования и решение задачи Римана о скачке // Алгебра и анализ. Тезисы докл. международной конференции, посвященной 100-летию Н. Г. Чеботарёва. Ч. ІІ. Казань, 1994. С. 96-97.

[18] Маркушевич А. И. Избранные главы теории аналитических функций. М.: Наука, 1976.

[19] Гамелин Т. Равномерные алгебры. М.: Мир, 1973.

[20] Долженко Е. П. О “стирании” особенностей аналитических функций // УМН. 1963. Т. 18. № 4. С. $135-142$. 